\title{
Cardiac magnetic resonance versus transthoracic echocardiography for the assessment of cardiac volumes and regional function after myocardial infarction: an intrasubject comparison using simultaneous intrasubject recordings
}

\author{
Blake I Gardner ${ }^{1,2}$, Scott E Bingham ${ }^{3}$, Marvin R Allen ${ }^{3}$, Duane D Blatter ${ }^{1}$ and \\ Jeffrey L Anderson*1,2
}

Address: ${ }^{1}$ Cardiovascular Department, Intermountain Medical Center, Intermountain Healthcare, 5121 South Cottonwood Street, Murray UT 84157, USA, ${ }^{2}$ University of Utah School of Medicine, 30 North 1900 East Room 1C109, Salt Lake City, Utah 84132, USA and ${ }^{3}$ Central Utah Clinic, 1055 North 500 West Suite 101, Provo, Utah 84604, USA

Email: Blake I Gardner - blake.gardner@imail.org; Scott E Bingham - sbingham@cut.net; Marvin R Allen - mallen@centralutahclinic.com; Duane D Blatter - duane.blatter@imail.org; Jeffrey L Anderson* - jeffrey.anderson@imail.org

* Corresponding author

Published: 18 August 2009

Cardiovascular Ultrasound 2009, 7:38 doi:10.1186/1476-7/20-7-38
Received: 24 March 2009

Accepted: 18 August 2009

This article is available from: http://www.cardiovascularultrasound.com/content/7/I/38

(c) 2009 Gardner et al; licensee BioMed Central Ltd.

This is an Open Access article distributed under the terms of the Creative Commons Attribution License (http://creativecommons.org/licenses/by/2.0), which permits unrestricted use, distribution, and reproduction in any medium, provided the original work is properly cited.

\begin{abstract}
Background: Although echocardiography is commonly used to evaluate cardiac function after MI, CMR may provide more accurate functional assessment but has not been adequately compared with echo. The primary study objective was to compare metrics of left ventricular volumes and global and regional function determined by cardiac magnetic resonance (CMR) and echocardiography (echo) in patients (pts) with recent myocardial infarction (MI).

Methods: To compare CMR with echo, 47 consecutive patients (pts 70\% male; mean age $=66 \pm$ II years) with MI $>6$ wks previously and scheduled for imaging evaluation were studied by both echo and CMR within $60 \mathrm{~min}$ of each other. Readers were blinded to pt information. Pearson's correlation coefficient, paired $t$-tests, and chi-square tests were used to compare CMR and echo measures. Further comparisons were made between pts and 30 normal controls for CMR and between pts and published normal ranges for echo.

Results: Measures of volume and function correlated moderately well between CMR and echo $(r=0.54$ to 0.75 , all $\mathrm{p}<0.00 \mathrm{I}$ ), but large and systematic differences were noted in absolute measurements. Echo underestimated left ventricular (LV) volumes (by $69 \mathrm{ml}$ for end-diastolic, $35 \mathrm{ml}$ for end-systolic volume, both $p<0.00 \mathrm{I}$ ), stroke volume (by $34 \mathrm{ml}, \mathrm{p}<0.00 \mathrm{I}$ ), and LV ejection fraction (LVEF) (by 4 percentage point, $p=0.02)$. CMR was much more sensitive to detection of segmental wall motion abnormalities $(p<$ $0.00 \mathrm{I}$ ). CMR comparisons with normal controls confirmed an increase in LV volumes, a decrease in LVEF, and preservation of stroke volume after MI.

Conclusion: This intra subject comparison after MI found large, systematic differences between CMR and echo measures of volumes, LVEF, and wall motion abnormality despite moderate inter-modality correlations, with echo underestimating each metric. CMR also provided superior detection and quantification of segmental function after MI. Serial studies of LV function in individual patients should use the same modality.
\end{abstract}




\section{Background}

Cardiac magnetic resonance (CMR) is increasingly utilized for dynamic imaging of the heart with the expectation that it will provide more accurate and reproducible measurements of cardiac chamber dimensions, volumes, and function compared to other non-invasive imaging techniques such as echocardiography and nuclear cardiography [1-3]. This expectation arises from the superior spatial resolution and more precise border definition achieved with CMR compared with these other techniques. However, the potential of CMR to be integrated optimally into clinical practice for assessment of cardiac metrics has been limited in part by a lack of comparative information with echocardiography (echo), the long-time clinical standard for non-invasive cardiovascular imaging, in health and disease. Myocardial infarction (MI) is the most common cardiovascular disease state routinely requiring imaging assessment. Little direct comparative information is available for these 2 techniques [4]. Thus, we undertook an intra subject comparison of echo with CMR in a consecutive cohort of post-MI patients to determine correlations and systematic differences between these modalities in assessing left ventricular volumes and function.

\section{Methods Study Objectives}

The main study objectives were: 1) to determine and compare metrics of left ventricular volumes and function between CMR and echo in a consecutive cohort of post-MI patients presenting for imaging evaluation of cardiac function, 2) to compare the sensitivity of the 2 modalities to assess regional wall motion (RWM) after MI, and 3) to quantitatively assess changes in these parameters after MI by a comparison of study patients with a cohort of normal volunteers.

\section{Study Population}

Forty-seven study subjects were prospectively enrolled from a consecutive series of patients with MI occurring $>6$ wks previously referred for echo evaluation of cardiac function and who consented to study participation, which included undergoing the complementary CMR study within 1 hour of echo. The protocol was approved by the Western Institutional Review Board. Standard electrocardiogram (ECG) and serial cardiac biomarker analysis was used to document MI.

Each subject served as his/her own control. Predefined cardiovascular measurements were made for CMR and echo using workstation-specific methods by a seasoned observer blinded to results of the complementary imaging method. Imaging results and demographic information were entered into a study database.
Given limited information on the normal range for CMR measurements and dependence of work-station specific methodology, we determined a normal range for CMR metrics in a group of 30 local normal volunteers recruited concurrently [5]. These subjects were consenting, uncompensated adults of either sex (equally divided between men and women) of ages between 40-60 years who underwent a standard CMR functional study and who had no clinically apparent cardiovascular diseases, including hypertension or diabetes on screening history, or abnormal physical on screening examination, or significant other-organ diseases. Echocardiographic normal values were taken from the published literature.

\section{Cardiac Magnetic Resonance Imaging (MRI) and Echo Studies}

Following axial and sagittal localizer sequences, standard cardiac 2, 3, and 4 chamber $1 \mathrm{~cm}$ thick long axis and short axis slices were obtained on a General Electric (GE) 1.5 Tesla magnet (EXCITE platform, version 11.0) using steady state free precession (SSFP) cine sequences (typical in-plane resolution $2.2 \times 1.3 \mathrm{~mm}^{2}$ ). All images were acquired using a phased-array 8-channel cardiac coil during single breath-holds (maximum, 15 seconds) with ECG (preferred) or peripheral (finger pulse) triggering. Acquisition parameters were as follows: zoom mode, TR = $2.4 \mathrm{msec}, \mathrm{TE}=\min$ full, flip angle $=45^{\circ}$, field of view $=35$ $\times 35 \mathrm{~cm}$, matrix $=192 \times 160$, NEX $=0.5$, slice thickness $=$ $8 \mathrm{~mm}$ with zero spacing, phases $=20$, and views per segment $\leq 24$ to maintain temporal resolution $<80 \mathrm{msec}$. No vasoactive agents were given between echocardiography and CMR imaging.

Echocardiographic images were obtained in the standard parasternal long and short axis and apical 4 chamber and 2 chamber views utilizing digital Vivid 7 ultrasound equipment with a combined tissue imaging $2.5-4.0 \mathrm{MHz}$ transducer (GE, Milwaukee, WI). At least 3 cardiac cycles were captured at the left ventricular (LV) base, mid papillary muscle level, and apex for wall motion assessment. No intravenous echocardiographic contrast agent was used. Later, an expert reader obtained measurements offline from the parasternal and apical windows blinded to patient identity, gender, and clinical data. Two dimensional (2D) echo ventricular volumes and LV ejection fraction were planimetered from the 4-chamber and 2chamber areas using the modified Simpson's rule. All of the measurements were obtained in concordance with American Society of Echocardiography standards [6].

\section{Image Analysis}

Off-line image post-processing was performed on a cardiac Delta workstation with ReportCard 2.0 software (GE, Waukesha, WI). Measurements followed standard CMR 
procedures or mimicked the approach to echo measurement where appropriate. Image grayscale was adjusted to maximize the myocardial blood pool contrast without maximal pixel intensity saturation. Manual planimetry was performed by an expert CMR technologist and independently confirmed by the CMR physician reader. (Observers were blinded to echo results.) In the short axis view, the most basal slice used for LV volume analysis excluded any LV outflow tract. Unattached papillary muscles were included within the left ventricular chamber. Volumes for CMR were determined from the stack of short axis slices using Simpson's rule and for echo using the modified, semi-Simpson's rule based on 4- and 2-chamber planimetry $[1,7,8]$. For both modalities, floating papillary muscles were included within the chamber volume measurements. For both modalities, wall segments were divided by consensus into 2 general categories: good/adequate and suboptimal/poor. To provide a conservative comparison of the 2 modalilties, poorly imaged segments were excluded from the comparative analyses.

Consensus quantitative results were entered prospectively into research databases, and computer-assisted manual tracings were saved for later visual comparisons. Similarly, echo image analysis was performed by experienced observers blinded to CMR study results and followed standard techniques [7-9].

Segmental wall motion analysis used a standard 17-segment model [10].

\section{Study Variables}

Study demographic variables included subject age, sex, heart rate, weight, height, and body surface area (BSA). Cardiovascular variables included LV end-diastolic (EDV) and end-systolic (ESV) volumes and the derived variables stroke volume (SV, SV = EDV-ESV), and LV ejection fraction $(\mathrm{EF}=\mathrm{SV} / \mathrm{EDV})$. For RWM, the LV was divided into 17 segments [11], and each visualized segment was graded on a scale of 1 to 5 as normal, hypokinetic, akinetic, or dyskinetic, or aneurysmal, respectively. Wall motion then was assessed as a summed total score, a worst segmental score, and an average RWM score, calculated as the average of all scored segments [7].

\section{Statistics}

Results are presented as mean (standard deviation). Pearson's correlation coefficient, paired $t$-tests, and chi-square tests were used to compare intra subject and inter modality CMR and echo metrics, as appropriate. SPSS for Windows (version 14.0, SPSS Inc., Chicago, IL) was used for statistical analysis. A p value of 0.05 or less was deemed nominally significant and 0.01 or less definitely significant, given the 5 primary outcome variables.

\section{Results}

\section{Subject Characteristics}

50 patients were initially enrolled. Three patients were excluded from study due to a rhythm other than sinus. Thus, the primary study population included 47 patients surviving MI that occurred at least 6 weeks previously. Demographics and selected patient characteristics are shown in Table 1 . Mean age averaged $66 \pm 11$ years; $70 \%$ of subjects were male.

The CMR control group included 30 healthy volunteers (15 women, 15 men) of average age 47 years (range, 40 to 60 ) with average BSA $1.7 \mathrm{sq} \mathrm{m}$ in women and $2.1 \mathrm{sq} \mathrm{m}$ in men. Study quality was excellent for all volunteers.

\section{CMR and Echo Volumetrics}

The volumetric measures of LVEDV, LVESV, and LVSV correlated moderately well between CMR and echo $(r=0.54$ to 0.75 , all $\mathrm{p}<0.001$ ), but large and systematic differences were noted in absolute measurements between the two (Table 2, Figure 1). Echo underestimated all 3 volumes: LVEDV by an average of $69 \mathrm{ml}$, LVESV by $35 \mathrm{ml}$, and stroke volume by $34 \mathrm{ml}$ (Table 2).

\section{Global and Segmental Function by CMR and Echo}

Global LV ejection fraction correlated moderately well between the 2 modalities $(\mathrm{r}=0.67, \mathrm{p}<0.001)$ (Table 2). However, echo underestimated LVEF compared to CMR by 4 percentage points $(\mathrm{p}=0.02)$.

All 799 segments in all 47 study patients could be scored by CMR, whereas $22(2.8 \%)$ segments $(\mathrm{p}<0.0001)$ in 9 patients $(19 \%)(\mathrm{p}=0.005)$ could not be adequately visualized to allow scoring by echo and are excluded from the comparative wall motion analyses with CMR.

In keeping with global function, segmental wall motion scores (WMS) showed moderately good correlations

Table I: Baseline Characteristics of the Post-MI Patient Cohort

\begin{tabular}{ll}
\hline Metric (unit) & Frequency/Descriptive \\
\hline N & 47 \\
Age: $y$, mean (SD) & $66(11)$ \\
Sex: \% (n) male & $70(33)$ \\
Heart rate: beats/min, mean (SD) & $64(14)$ \\
Body surface area: m $^{2}$, mean (SD) & $1.96(0.23)$ \\
Location of WMA $10 *:$ & $62(29)$ \\
$\quad$ Anterior/Anteroseptalt, \% (n) & $77(36)$ \\
Inferiort, \% (n) & $45(21)$ \\
Lateralt, \% (n) & $68(32)$ \\
Apicalt, \% (n) & $4(2)$ \\
Nonet, \% (n) &
\end{tabular}

*Wall motion abnormality (WMA) assessed by CMR using the standard 17 segment model with assigned coronary territories ${ }^{10} \dagger$ 


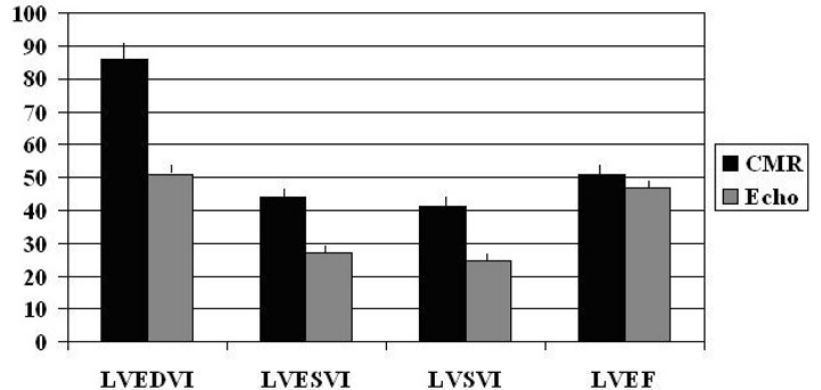

Figure I

LV Volumes (Indexed to BSA) and Function by CMR versus Echo. Abbreviations: $C M R=$ cardiac magnetic resonance; echo = echocardiography. Left ventricular end diastolic volume index (LVEDVI), left ventricular end systolic volume index LVESVI), left ventricular stroke volume index (LVSVI), left ventricular ejection fraction (LVEF). Volumes are indexed to body surface area (BSA). Bars represent means, given with standard error whiskers. Comparisons of LVEDVI, LVESVI, and LVSVI between CMR and echo are significant at $p<0.001$, and LVEF at $p=0.02$.

between CMR and echo, both for the worst WMS ( $\mathrm{r}=$ $0.69, \mathrm{p}<0.001)$ and for total and average segmental WMS $(\mathrm{r}=0.66, \mathrm{p}=0.001)$ (Table 2). However, echo underestimated WMS of the worst segment (by 0.81 grade, $\mathrm{p}<$ $0.001)$, as well as the average WMS and the summed WMS compared to CMR (both $\mathrm{p}<0.001$ )(Table 2). Wall motion was completely normal in 15 patients $(32 \%)$ assessed by echo, compared to only 2 (4\%) assessed by CMR ( $\mathrm{p}<0.001)$, suggesting that CMR is more sensitive in distinguishing diseased from normal subjects.

\section{Subgroup Analysis by Degree of LV Dysfunction}

To see whether differences in metrics by modality are affected by degree of LV dysfunction, comparisons were made between subgroups of patients with less than versus more than or equal to the median echo LVEF of $46 \%$. For both modalities, LVEDV and LVESV increased in the lower
LVEF subgroup, whereas LVSV was unchanged. However, inter modality differences in paired CMR versus echo LVEF and volumetrics were not significantly dependent on LVEF subgroup (i.e., mean $\triangle \mathrm{LVEF}, 1.4 \%$ vs. $4.8 \%$, $\mathrm{p}=$ 0.2 ; mean $\triangle$ LVEDV $58 \mathrm{ml}$ vs. $77 \mathrm{ml}, \mathrm{p}=0.15$; high vs. low LVEF subgroups, respectively).

\section{Post-MI Patients and Normals Compared by CMR}

Table 3 compares the primary volumetric and functional metrics, determined by CMR, between the post-MI patient cohort and normal volunteers, indexed to BSA. Stroke volume is preserved but is associated with moderate decreases in LVEF accompanied by moderate to large increases in LVEDVI and LVESVI

\section{Post-MI Patients and Normals Compared by Echo}

Table 4 compares the primary volumetric and functional metrics, determined by echo, between the post-MI patient cohort and a report of normal values. LVEF is moderately decreased compared to normals. LVEDV and LVESV differences from normal values are modest and overlapping.

Indirect Comparison of Normal Ranges for CMR vs. Echo As with the intra subject comparisons of CMR and echo in post-MI patients, an indirect comparison of the normal ranges for volunteers in our study and with a published normal range for echocardiographic metrics also suggests important differences in volumes (Table 3 vs. 4) [12].

\section{Discussion}

\section{Study Overview}

We performed a moderately large comparative CMR and echo imaging study of post-MI patients in which each subject served as his or her own control. We found that metrics of LV volumes and function, acquired from near simultaneous studies, while correlating moderately well, showed large and systematic inter-modality differences. Echo underestimated all primary measures compared with CMR, including LVEDV, LVESV, SV, LVEF, and, importantly, RWM abnormality. CMR also was more sensitive in distinguishing diseased from normal subjects. Of

Table 2: Summary of LV Volumes and Function by CMR versus Echo in the Post-MI cohort: Means (SD)

\begin{tabular}{|c|c|c|c|c|c|}
\hline Metric (unit) & CMR & Echo & $\mathrm{p}$-value & Correlation(r) & P-value \\
\hline LV EDV (ml) & $171(62)$ & $102(42)$ & $<0.001$ & 0.701 & $<0.001$ \\
\hline LV ESV (ml) & $88(47)$ & $53(28)$ & $<0.001$ & 0.746 & $<0.001$ \\
\hline LV SV (ml) & $83(24)$ & $49(22)$ & $<0.001$ & 0.536 & $<0.001$ \\
\hline LV EF (\%) & $51(1 \mathrm{I})$ & 47 (II) & 0.02 & 0.672 & $<0.001$ \\
\hline Worst WMS (units) & $2.79(0.88)$ & $1.98(0.85)$ & $<0.001$ & 0.692 & $<0.001$ \\
\hline Total WMS/pt & $26.0(7.0)$ & $23.0(7.0)$ & 0.001 & 0.657 & $<0.001$ \\
\hline Average WMS & $\mathrm{I} .53(0.4 \mathrm{I})$ & $1.39(0.42)$ & 0.001 & 0.657 & $<0.001$ \\
\hline
\end{tabular}

Abbreviations: $C M R$ = cardiac magnetic resonance; echo = echocardiography; $\mathrm{Ml}=$ myocardial infarction. Left ventricular end diastolic volume (LVEDV), left ventricular end systolic volume (LVESV), left ventricular stroke volume (LVSV), left ventricular ejection fraction (LVEF), I7 segment wall motion score (WMS) 
clinical importance, these differences by modality suggest that serial measurements of function should be performed using the same method, as large and spurious differences may be seen if modalities are switched. Finally, given its more accurate and reproducible determination of function, CMR should be preferred when small to moderate serial changes in these functional parameters are of clinical importance to disease management.

\section{Previous Work and Study Rationale}

Echocardiography is a mature imaging modality $[13,14]$ with an accepted if not extensively studied normal range for ventricular volumes $[7,12,15,16]$. Normal values for contemporary CMR also are now published [5,17-20]. Indirect and direct comparisons of these studies suggest that echocardiographic normal values may not accurately reflect the normal range for ventricular $[4,7,19]$ or atrial [21] chamber measurements by CMR. A study in which each imaging modality is tested (near) simultaneously within the same subjects, and which uses carefully standardized techniques and blinded assessment, represents the most accurate and informative design to compare imaging modalities. Because coronary artery disease represents the most common reason for applying these imaging tests, we chose a typical post-MI population for a direct, intra subject comparative study of these 2 modalities.

Maciera and Royal Brompton Hospital (London) collaborators have reported on normalized left ventricular systolic and diastolic function by CMR in a moderately large study sample $(\mathrm{n}=120)$ using contemporary scanner $(1.5$ T) and sequence (SSFP) techniques [17]. CMR analysis used a computer-based technology with blood pool thresholding to delineate the papillary muscles, which were excluded from chamber volume and included in mass measurements. As might be expected, mass measurements by this method were larger and volume measurements smaller than results obtained with the operatorinteractive inclusion method used here, emphasizing the need for method-specific normal values. Alfakih also has

Table 3: Comparison of Metrics in Post-MI versus Normal Subjects by CMR Indexed to Body Surface Area*: Means (SD)

\begin{tabular}{llll}
\hline Metric (unit) & Post-MI $(\mathrm{N}=47)$ & Normals $(\mathrm{N}=30)$ & P-value \\
\hline LV EDVI* $\left(\mathrm{ml} / \mathrm{m}^{2}\right)$ & $86(28)$ & $68(10)$ & 0.004 \\
LV ESVI* $\left(\mathrm{ml} / \mathrm{m}^{2}\right)$ & $44(22)$ & $25(7.0)$ & $<0.001$ \\
LV SVI* $\left(\mathrm{ml} / \mathrm{m}^{2}\right)$ & $4 I(I I)$ & $42(5.0)$ & 0.72 \\
LV EF $(\%)$ & $5 I(1 \mathrm{l})$ & $65(6.0)$ & $<0.00 \mathrm{I}$
\end{tabular}

\section{*ANOVA adjusted for sex.}

Abbreviations: $C M R=$ cardiac magnetic resonance. $\mathrm{MI}=$ myocardial infarction. Left ventricular end diastolic volume index (LVEDVI), left ventricular end systolic volume index (LVESVI), left ventricular stroke volume index (LVSVI), left ventricular ejection fraction (LVEF)
Table 4: Comparison of Metrics in Post-MI Patients versus Normal Subjects by Echo Indexed to Body Surface Area*: Means (SD)

\begin{tabular}{lll}
\hline Metric (unit) & Post-MI $(\mathbf{N}=47)$ & Normals ${ }^{12 *}$ \\
\hline LV EDVI* $\left(\mathrm{ml} / \mathrm{m}^{2}\right)$ & $5 I(19)$ & $54.5(9)$ \\
$\operatorname{LV~ESVI*~}\left(\mathrm{ml} / \mathrm{m}^{2}\right)$ & $27(13)$ & $22(5)$ \\
$\operatorname{LV~SVI}\left(\mathrm{ml} / \mathrm{m}^{2}\right)$ & $25(12)$ & $22.5 \dagger$ \\
$\operatorname{LV~EF~}(\%)$ & $47(11)$ & $60(6)$ \\
\hline
\end{tabular}

†Derived estimate

Abbreviations: $\mathrm{MI}=$ myocardial infarction. Echo = echocardiography. Left ventricular end diastolic volume index (LVEDVI), left ventricular end systolic volume index (LVSVI), left ventricular stroke volume index (LVESVI), left ventricular ejection fraction (LVEF)

reported normal ranges for CMR using SSFP sequences [18], but some of the same issues limit the comparison of their results with the normal values derived for the present study [5].

Normal values for cardiovascular function by MRI also were recently assessed in 800 adult participants, equally representing 4 ethnic groups, both sexes, and 4 age decades, in the multi-ethnic study of atherosclerosis [19]. As in our study, papillary muscles were included in LV volume and were excluded from LV mass calculations. Results were generally similarly to those in our population reported here for $\mathrm{LV}$ volumes and ejection fraction.

Little previous information is available directly comparing these two modalities in coronary artery disease. Jenkins et-al published a comparative study in 50 patients with previous MI [4]. Patients with poor echo images were excluded. Correlations of MRI versus echo for LVEDV, LVESV, and LVEF were generally similar (i.e., $r=0.61$ to $0.81, \mathrm{p}<0.01$ ) to those we observed (Table 2). LV volumes were slightly larger and LVEF lower in their report, indicating a somewhat sicker population. Also consistent with our observations, volumes and LVEF were under estimated by two-dimensional echo. Their study also assessed incremental change after MI over a 1-year interval, but, unlike our study, it did not assess or compare regional wall motion between modalities.

\section{Explanatory Mechanisms}

This study did not directly determine the reasons for underestimations of volumes by echo compared with CMR. However, lower spatial (and temporal) resolution of echo, especially at far field, together with suboptimal acoustic windows in some patients with less complete visualization of wall segments, and greater "blooming" of lumen/wall boundaries with echo are disadvantages relative to CMR and likely contributors to observed differences. 


\section{Clinical Implications}

Quantitative measurements of cardiovascular anatomy and function are only of use if they are accurate and reproducible and if they can be compared with a normal, expected range of values. Incorrect interpretations, based on an incorrect comparative range of normal, large re-test variability, or spurious differences on serial testing with mixed modalities can lead to inappropriate disease assessment and management. Thus, the positive impact on clinical practice of accurate, reproducible measures of LV volumes and segmental and global function cannot be over emphasized. Despite these advantages of CMR, echocardiography has been well demonstrated by large and multicenter studies to be effective in evaluating and stratifying patients after MI with LV dysfunction. The clinical database for CMR, although considered a gold standard, is still substantially smaller. The choice of an imaging option should take cost as well as technical comparisons into account to achieve an optimal cost-effectiveness result. CMR is a moderately more expensive modality: our current Medicare facility/professional reimbursement rates for non-contrast studies are USD \$679/121 (CMR) versus $\$ 412 / 70$ (echo), with full price charges often significantly higher. Beyond this, CMR requires moderately increased acquisition and analysis times and greater equipment and facility expenditures.

\section{Study Strengths and Limitations}

The study presents a direct comparison of imaging modalities in a primary study population sample of coronary artery disease patients, for whom imaging was clinically indicated. Only one study was obtained for each modality, so that re-test variability could not be tested. Similarly, measurements were arrived at by consensus, so that inter observer variability cannot be assessed. Other published reports provide some information on these measures $[4,5,19]$. Although slight differences in measurement methodology (Simpson's vs. semi-Simpson's) could contribute to results, study methods represent those most commonly used and validated in practice for each modality. Also, given close intra-modality correlations between these 2 methods, any differences due to measurement methodology are likely to be very small. Contrast echocardiography and three dimensional (3-D) echocardiography may provide more accurate LV volumetric and functional measures than standard 2D echocardiography, performed here, and may also correlate better with CMR [4]. (It should be noted that 3-D CMR also is becoming available and also may improve CMR slice selection.) However, these techniques may add time and expense to standard 2D techniques and are not fully incorporated into routine clinical practice. The study is of only moderate size and in a specific population, although size was adequate to demonstrate systematic differences in the studied metrics. Cost-effectiveness considerations also must be considered in clinical practice but are not specifically addressed by this study. Finally, the reference control group could be studied by CMR only.

\section{Conclusion}

In a moderately large CMR versus echocardiographic comparative study in post-MI patients in which each patient served as his or her own control, metrics of LV volume and function, while moderately correlated, showed significant systematic inter modality differences, with echo underestimating all 5 primary measurement variables. CMR also was more sensitive in distinguishing wall motion abnormalities. Of clinical importance, these differences by modality suggest that serial measurements of function should be performed using the same method. Given its greater accuracy and reproducibility, CMR may be preferred when small to moderate serial changes in these metrics are clinically important.

\section{Competing interests}

The authors declare that they have no competing interests.

\section{Authors' contributions}

BIG participated in study planning and design, performed data analysis, and helped to draft the manuscript. SEB participated in study planning and design, took part in data acquisition, performed data analysis, and assisted with manuscript revision. MRA took part in data acquisition, performed data analysis, and assisted with manuscript revision. DDB took part in data acquisition, performed data analysis, and assisted with manuscript revision. JLA participated in study planning and design, took part in data acquisition, performed data analysis, helped to draft the manuscript, and assisted with manuscript revision. All authors read and approved the final manuscript.

\section{Acknowledgements/Financial Support}

This study was self-funded by the Departments of Cardiovascular Medicine, LDS Hospital, Intermountain Healthcare, Salt Lake City and Central Utah Clinic, Provo, Utah.

\section{References}

I. Manning WJ, Pennell DJ: Cardiovascular Magnetic Resonance New York: Churchill Livingston; 2002.

2. Grothues F, Smith GC, Moon IC, Bellenger NG, Collins P, Klein HU, Pennell DJ: Comparison of interstudy reproducibility of cardiovascular magnetic resonance with two-dimensional echocardiography in normal subjects and in patients with heart failure or left ventricular hypertrophy. Am J Cardiol 2002, 90:29-34.

3. Pohost GM, Hung L, Doyle M: Clinical use of cardiovascular magnetic resonance. Circulation 2003, 108:647-653.

4. Jenkins C, Bricknell K, Chan J, Hanekom L, Marwick TH: Comparison of two- and three-dimensional echocardiography with sequential magnetic resonance imaging for evaluating left ventricular volume and ejection fraction over time in patients with healed myocardial infarction. Am J Cardiol 2007, 99:300-306.

5. Anderson JL, Weaver AN, Horne BD, Jones HU, Jelaco GK, Cha JA, Busto HE, Hall J, Walker K, Blatter DD: Normal cardiac magnetic resonance measurements and causes of interobserver dis- 
crepancies in volumes and mass using the papillary muscle inclusion method. Open General and Internal Medicine Journal 2007, I:6-12.

6. Gardin JM, Adams DB, Douglas PS, Feigenbaum $H$, Forst DH, Fraser AG, Grayburn PA, Katz AS, Keller AM, Kerber RE, Khandheria BK, Klein AL, Lang RM, Pierard LA, Quinones MA, Schnittger I, American Society of Echocardiography: Recommendations for a standardized report for adult transthoracic echocardiography: a report from the American Society of Echocardiography's Nomenclature and Standards Committee and Task Force for a Standardized Echocardiography Report. J Am Soc Echocardiogr 2002, 15:275-290.

7. Feigenbaum H: Echocardiographic measurements and normal values. In Echocardiography 5th edition. Edited by: Feigenbaum $\mathrm{H}$. Philadelphia: Lea and Febiger; 1994:658-695.

8. Reynolds T: 2-D numbers at a glance. In Echocardiographer's pocket reference Phoenix: Arizona Heart Institute Foundation. School of Cardiac Ultrasound; 2000:166.

9. Gerstenblith G, Frederiksen J, Yin FC, Fortuin NJ, Lakatta EG, Weisfeldt ML: Echocardiographic assessment of a normal adult aging population. Circulation 1977, 56:273-278.

10. Cerqueira M: Standardized myocardial segmentation and nomenclature for tomographic imaging of the heart: a statement for healthcare professionals from the Cardiac Imaging Committee of the Council on Clinical Cardiology of the American Heart Association. Circulation 2002, 105:539-542.

II. Bourdillon PD, Broderick TM, Sawada SG, Armstrong WF, Ryan T, Dillon JC, Fineberg NS, Feigenbaum H: Regional wall motion index for infarct and noninfarct regions after reperfusion in acute myocardial infarction: comparison with global wall motion index. J Am Soc Echocardiogr 1989, 2:398-407.

12. Gordon EP, Schnittger I, Fitzgerald PJ, Williams P, Popp RL: Reproducibility of left ventricular volumes by two-dimensional echocardiography. J Am Coll Cardiol I983, 2:506-5I3.

13. Cheitlin MD, Armstrong WF, Aurigemma GP, Beller GA, Bierman FZ, Davis JL, Douglas PS, Faxon DP, Gillam LD, Kimball TR, Kussmaul WG, Pearlman AS, Philbrick JT, Rakowski H, Thys DM, Antman EM, Smith SC Jr, Alpert JS, Gregoratos G, Anderson JL, Hiratzka LF, Hunt SA, Fuster V, Jacobs AK, Gibbons RJ, Russell RO, American College of Cardiology; American Heart Association; American Society of Echocardiography: ACC/AHA/ASE 2003 guideline update for the clinical application of echocardiography: summary article: a report of the American College of Cardiology/American Heart Association Task Force on Practice Guidelines (ACC/AHA/ASE Committee to Update the 1997 Guidelines for the Clinical Application of Echocardiography). Circulation 2003, 108: I| |46-I I62.

14. Feigenbaum H, Armstrong WF, Ryan T: Feigenbaum's echocardiography 6th edition. Phildelphia: Lippinctoo Williams \& Wilkins; 2005.

15. Erbel R, Schweizer P, Herrn G, Mayer J, Effert S: Apical two-dimensional echocardiography: normal values for single and biplane determination of left ventricular volume and ejection fraction. Dtsch Med Wocheschr 1982, 107:1872-1877.

16. Reynolds T: The echocardiographer's pocket reference Phoenix: Arizona Heart Institute; 2000.

17. Maceira AM, Prasad SK, Khan M, Pennell DJ: Normalized left ventricular systolic and diastolic function by steady state free precession cardiovascular magnetic resonance. J Cardiovasc Magn Reson 2006, 8:417-426.

18. Alfakih K, Plein S, Thiele H, Jones T, Ridgway JP, Sivananthan MU: Normal human left and right ventricular dimensions for MRI as assessed by turbo gradient echo and steady-state free precession imaging sequences. J Magn Reson Imaging 2003, 17:323-329.

19. Natori S, Lai S, Finn JP, Gomes AS, Hundley WG, Jerosch-Herold M, Pearson G, Sinha S, Arai A, Lima JA, Bluemke DA: Cardiovascular function in multi-ethnic study of atherosclerosis: normal values by age, sex, and ethnicity. Am J Roentgenol 2006, $186(6$ Suppl 2):S357-S365.

20. Lorenz CH, Walker ES, Morgan VL, Klein SS, Graham TP: Normal human right and left ventricular mass, systolic function and gender differences by cine magnetic resonance imaging. J Cardiovasc Magn Reson 1999, I:7-21.

21. Anderson JL, Horne BD, Pennell DJ: Atrial Dimensions in Health and Left Ventricular Disease Using Cardiovascular Magnetic Resonance. J Cardiovasc Magn Reson 2005, 7:67I-675.
Publish with Biomed Central and every scientist can read your work free of charge

"BioMed Central will be the most significant development for disseminating the results of biomedical research in our lifetime. "

Sir Paul Nurse, Cancer Research UK

Your research papers will be:

- available free of charge to the entire biomedical community

- peer reviewed and published immediately upon acceptance

- cited in PubMed and archived on PubMed Central

- yours - you keep the copyright

Submit your manuscript here:

http://www.biomedcentral.com/info/publishing_adv.asp
BioMedcentral 